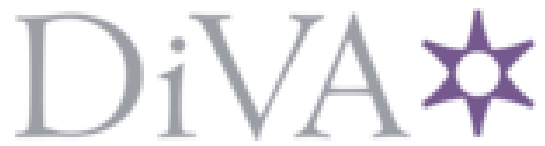

http://www.diva-portal.org

Preprint

This is the submitted version of a chapter published in High-Throughput Glycomics and Glycoproteomics: Methods and Protocols.

Citation for the original published chapter:

Lisacek, F., Mariethoz, J., Alocci, D., Rudd, P M., Abrahams, J L. et al. (2017)

Databases and Associated Tools for Glycomics and Glycoproteomics

In: Gordan Lauc, Manfred Wuhrer (ed.), High-Throughput Glycomics and

Glycoproteomics: Methods and Protocols (pp. 235-264). Humana Press

Methods in Molecular Biology

https://doi.org/10.1007/978-1-4939-6493-2_18

N.B. When citing this work, cite the original published chapter.

Permanent link to this version:

http://urn.kb.se/resolve?urn=urn:nbn:se:su:diva-184079 


\title{
Chapter 21. Databases and associated tools for glycomics and glycoproteomics
} Frederique Lisacek ${ }^{1}$, Julien Mariethoz ${ }^{1}$, Lou Gotz ${ }^{1}$, Pauline M. Rudd ${ }^{2}$, Jodie L. Abrahams ${ }^{3}$, Matthew P. Campbell ${ }^{3}$, Nicolle H. Packer ${ }^{3}$, Jonas Ståhle ${ }^{4}$, Göran Widmalm ${ }^{4}$, Barbara V. Adamczyk $^{5}$, Miguel S. Rojas Macias ${ }^{5}$, Chunsheng Jin $^{5}$ and Niclas G. Karlsson ${ }^{5}$

${ }^{1}$ Proteome Informatics Group, SIB Swiss Institute of Bioinformatics, Geneva, Switzerland ${ }^{2}$ NIBRT GlycoScience Group, NIBRT - The National Institute for Bioprocessing Research and Training, Fosters Avenue, Mount Merrion, Blackrock, Co. Dublin, Ireland ${ }^{3}$ Biomolecular Frontiers Research Centre, Macquarie University, North Ryde, NSW, Australia

${ }^{4}$ Department of Organic Chemistry, Arrhenius Laboratory, Stockholm University, Sweden.

${ }^{5}$ Department of Medical Biochemistry and Cell Biology, Institute of Biomedicine, Sahlgrenska Academy, University of Gothenburg, Gothenburg, Sweden

\begin{abstract}
The access to biodatabases for glycomics and glycoproteomics has proven to be essential for current glycobiological research. This chapter presents available databases that are devoted to different aspects of glycobioinformatics. This includes oligosaccharide sequence databases, experimental databases, 3D structure databases (of both glycans and glycorelated proteins) and association of glycans with tissue, disease and proteins. Specific search protocols are also provided using tools associated with experimental databases for converting primary glycoanalytical data to glycan structural information. In particular, researchers using glycoanalysis methods of HPLC (GlycoBase), MS (GlycoWorkbench, UniCarb-DB, GlycoDigest) and ${ }^{1} \mathrm{H}$ NMR (Casper) will benefit from this chapter. In addition we also include information on how to utilise glycan structural information to query databases that associate glycans with proteins (UniCarbKB) and with interactions with pathogens (SugarBind)

\section{ii. Key Words}

Glycobioinformatics, Oligosaccharide, Omics, HPLC, NMR, Mass spectrometry, Databases
\end{abstract}




\section{Introduction}

The success of bioinformatics in providing breakthroughs in life science research has been gradual and it is hard to say when it all began. Publically accessible life science databases, with their symbiotic connection to the open internet, turned the concept into reality at the end of last century. At the same time, the need to query databases with intelligent software increased. Genomic databases are currently the dominating resource, and the population of these databases with genome sequences has been boosted by analytical developments starting from Sanger sequencing to what is now known as NextGen sequencing that allows sequencing of the genome (DNA) and transcriptome (mRNA). These nucleotide databases (Genebank, European Nucleotide Archive and DNA Data Bank of Japan) are now invaluable resources for the life sciences. Subsequently, with the improvement in biological mass spectrometric ionization techniques such as ESI and MALDI, large scale protein identification (proteomics), utilizing nucleotide databases, became possible. Other -omics such as metabolomics and, as is the case for this chapter, glycomics/glycoproteomics has been more challenging because the molecules are secondary gene products that cannot be predicted from the genomic databases. Instead, the generation of glycan structural databases relies on data generated from the glycoanalytical field, and on the development of glycobioinformatics to allow the transition from glycocommunity to the wider life science arena. Early attempts to provide glycostructural databases include CarBank (ref), EuroCarb (ref) and GlycoSuiteDB (ref), and their legacy whereby they are now integrated into the UniCarb family (ref), GlycomeDB (ref), GlycoBase (ref) and GRITS (ref). With the realisation that understanding post-translational modifications is key to describing the functional molecules that operate in every cell in an the organism, glycodatabases and associated tools have become a prerequisite for life science research, not only for addressing glycobiology specifically, but for overall understanding of cell systems biology. Eventually the integration of various -omics data will be required for modulating an in-silico cell (Figure 1).

The type of databases that are available can roughly be divided into analytical databases that can be queried and compared with data to predict structures, and databases that can be queried by the resultant glycan structural information to obtain associated metadata (including binding partners, associated glycosyltransferases and glycosidases, attached proteins, lipids, related organisms and diseases, prediction of 3D structures). Glycosylation information can also be found in more generic life science databases such as UniProt, Protein DataBase, Protein Atlas. Without claiming to describe every resource that is available we provide a table (Table 1) containing many glycodatabases. In addition we will also describe in detail a selection of tools that can be used to interpret glycomic data generated by some of the more wellestablished analytical methods, including MS, H-NMR and HPLC. The focus of the chapter is towards the application of bioinformatics to the $N$-linked and $O$-linked glycans on mammalian glycoproteins. This chapter also includes examples of how to indicate how glycan structures interacts with pathogens and for finding proteins associated with a particular glycan structure(s). The methods described here are devoted to interpretation of data generated by glycomic/glycoproteomic analysis. There are other chapters and literature that specifically address the workflows for how to generate data that can be used for the tools presented in this chapter (ref Catherine). We have included datasets as supplementary material to this chapter for the reader to test the different query functions of the databases and the tools. 


\section{Materials}

\subsection{From analytical MS data to monosaccharide composition: GlycoMod}

With GlycoMod it is possible to predict the monosaccharide composition of free or derivatized $N$ - and $O$-glycans released from glycoproteins (Note 1 ), as well as the monosaccharide composition on glycopeptides from proteolytically digested proteins (using a submitted peptide or protein sequence), from experimentally determined MS data. The prediction is based on calculation of the combination of monosaccharide masses with some rules restricting unlikely combinations.

On-line web site: web.expasy.org/glycomod/

Type of data: MS peak list can be submitted from both negative and positive ion mode mass spectrometry.

Test data for:

$N$-linked glycopeptides: $N$-linked glycopeptide of human alpha-HS-glycoprotein (P02765), reduced and alkylated with iodoacetamide with an $[\mathrm{M}+\mathrm{H}]^{+}$ion of $\mathrm{m} / \mathrm{z} 3394.4495(+/-0.05$

$\mathrm{Da})$

Reduced, released $O$-linked oligosaccharides: $[\mathrm{M}-\mathrm{H}]^{-}$ions of $m / z(+/-0.3 \mathrm{Da})$

Released AB-labelled $N$-linked glycans: $[\mathrm{M}-\mathrm{H}]^{-}$ions of $\mathrm{m} / \mathrm{z}$ 1581.92, 1744.00 and 1906.01 (+/- 0.5) mass of 2-aminobenzamide 136.06 Da

\subsection{From structure to predicted MS/MS data: GlycoWorkbench}

GlycoWorkbench is designed to facilitate the annotation of mass spectrometry data as well as presenting corresponding glycan structures (ref Ceroni, A. et al., 2008, Journal of Proteome Research, 7:1650; Damerell, D., 2012, Biol Chem, 393:1357). A particular feature of this software is that it provides a friendly interface to draw various glycans (different types, labelling, and charge states). The theoretical MS/MS fragment of selected structure can be computed.

Website for downloading tool: code.google.com/p/glycoworkbench/

Type of data: MS/MS peak lists of glycans

Test MS/MS data: A peak list of a PA-labelled $N$-linked glycan with [M-H] ${ }^{-}$ions of $\mathrm{m} / \mathrm{z}$ 1311.08 and a omposition of 5 hexoses and $2 N$-acetylhexosamines (Supplementary Table 1 )

\subsection{From analytical MS and MS/MS data to structure: UniCarb-DB}

UniCarb-DB (ref) provides access to a collection of LC-MS/MS $N$ - and $O$-linked glycan fragments released from glycoproteins and complemented with experimental metadata. UniCarb-DB supports the comparison of users experimental spectra against the experimental spectra in the database. Therefore, UniCarb-DB can be used as reference to aid during manual annotation and development of more precise software tools to analyse and interpret spectrometric data.

On-line website: www.unicarb-db.org

Type of data: MS/MS fragment peak list from bar spectra together with pseudomolecular ion mass (Note 2) 
Test MS data for MS/MS search: MS/MS peak lists of a reduced $N$-linked oligosaccharide with of $[\mathrm{M}-\mathrm{H}]$ ion of $\mathrm{m} / \mathrm{z} 1056.48$ (Supplementary Table 2)

\subsection{From H-NMR data to structure: Casper}

The computer program CASPER (Computer Assisted SPectrum Evaluation of Regular polysaccharides) was originally developed to facilitate structural elucidation of polysaccharides using as input sugar and methylation analyses together with unassigned ${ }^{13} \mathrm{C}$ NMR data [ref CR 1987]. Subsequently, ${ }^{1} \mathrm{H}$ and 2D NMR data were made possible as input to the program [PMID:2673508, PMID:1757506, PMID:9691437], also for oligosaccharides [PMID:1807153], in the analysis of unknown structures. CASPER is used in two modes: (i) prediction of ${ }^{1} \mathrm{H}$ and ${ }^{13} \mathrm{C}$ NMR chemical shifts for a given structure as input or (ii) structural determination of a glycan using unassigned ${ }^{1} \mathrm{H}$ and/or ${ }^{13} \mathrm{C}$ NMR data as well as components [PMID:21280662, PMID:21955217] and linkage positions when known, e.g. as suggested by bioinformatics information in the database ECODAB [PMID:19926726, PMID:25352573]. Besides NMR chemical shift predictions and structural elucidation based on the sugar residues substituents (eg $O$-acetyl and phosphates) can be added as well as reducing end modifications [PMID:21621752, PMID:24036391].

On-line website: www.casper.organ.su.se/casper/ [PMID:16564037].

\subsection{From HPLC/UPLC/CE data to $N$-linked structure: GlycoBase}

GlycoBase contains tools that allow users to conduct structural assignments using matching retention times to a standard dextran ladder (GU) values. Further confirmation of structures using on line mass spectrometry, exoglycosidase digestions followed by further chromatographic analysis can be performed to determine monosaccharide sequence and linkage.

On-line open access website: glycobase.nibrt.ie

Type of data: Chromatographic data: 2-aminobenzamide labeled released glycans with GU values assigned from and relative \% areas. Description of how data can be generated is found in in Chapter 4 and Chapter 10 and in references [1-3]. Test data can be found in the table in Figure 7. APTS labelled glycans can be run on CE using the same rationale.

\subsection{Validation of monosaccharide sequence and linkages with the aid of exoglycosidase digestion: GlycoDigest}

Description of database/tool

One of the most commonly used methods for determining the sequence and structure of oligosaccharides is the enzymatic analysis of oligosaccharides using highly specific exoglycosidases, either sequentially or in array form. GlycoDigest is a web-application and standalone tool that simulates the behaviour of these exoglycosidase digestions on released $\mathrm{N}$ and O-linked oligosaccharides. The tool is based on a set of rules acquired from expert knowledge and supporting experimental evidence provided by GlycoBase, and can be used to tailor a panel of exoglycosidases to unambiguously confirm the structure of oligosaccharides. On-line website: www.glycodigest.org

Type of data: A description of the enzymes supported and a user guide are can be accessed at www.glycodigest.org and in reference (PMID: 


\subsection{Literature report on structure and association with protein: UniCarbKB}

UniCarbKB is an international collaboration to develop an open source glycobioinformatics database to support glycomics and the emerging technology of glycoproteomics (PMID 24234447). It is a curated collection of information on well-characterised glycan structures derived from glycoproteins, biological fluids and tissues. Although UniCarbKB provides annotated entries for all species, its primary focus is the annotation of proteins from mammalian systems of distinct taxonomic groups to ensure the presence of high quality annotations for representative members of all protein families. The database provides contextual information for $\mathrm{N}$ - and $\mathrm{O}$-glycan structures attached to proteins and where known, maintains the commonly lost connection between a glycan structure and the attached proteins as annotated in UniProtKB and neXtProt. In addition, entries are supplemented with descriptions of their biological source, supporting reference and the experimental methods employed to determine the glycan structure.

On-line web site: www.unicarbkb.org

\subsection{Association of structure with microbial interactions: SugarBind}

Description of database/tool

On-line web site: http://sugarbind.expasy.org/

Frederique and Julien (also 3.8)

\section{Methods}

\subsection{From analytical MS data to monosaccharide composition: GlycoMod}

The most straightforward use of MS analysis for glycoconjugates is to determine monosaccharide compositions based on experimental masses, and GlycoMod can be used for this purpose on both released glycans and glycopeptide MS data. GlycoMod is also crosslinked to UniCarbKB, where predicted compositions are linked to possible glycan sequences reported in the literature and found in the database, allowing the combinatorial possibilities of monosaccharides to be narrowed down to previously seen and reported structures in biological systems.

\subsubsection{Searching GlycoMod using oligosaccharides and glycopeptides}

1. Launch the GlycoMod website

2. Export peaklists of singly charged pseudomolecular ions from mass spectrometric software (Note 3).

3. Paste the peaklist of pseudomolecular ions (1.) together with information of type of adducts and ion mode (2.) and MS accuracy (3.) into the website (Figure 2A) (see test data in 2.1).

4. Provide information into the website about the type of glycoconjugate you are analysing:
a) $\mathrm{N}$ - or $\mathrm{O}$-linked 
b) Glycopeptides, free oligosaccharides, reduced oligosaccharides (4.) (for the $O$-linked test data) and derivatised oligosaccharides (for the $N$-linked test data)

c) Glycoprotein sequence or UniProt ID, type of protease, and cysteine modification (5) (for glycopeptide test data insert P02765, trypsin and iodoacetamide, respectively)

d) Reducing end derivatization mass (6.) (for the $N$-linked test data 136.06)

e) Type of monosaccharides (7.) (Note 4)

5. Submit the search

6. The results are displayed with each submitted experimental match with the selection of different compositions matching your data. The reference to the UniCarbKB database can be used as a first indicator to narrow down the possibilities. The data will be provided as compositions and MS error and glycoforms mass (Note 5).

7. For the glycopeptide data the result should be: 1736.65 (glycoform mass), -0.003 $(\Delta \text { mass (Dalton)), (HexNAc) } 2 \text { (Deoxyhexose) })_{3}+(\text { Man)3(GlcNAc) })_{2}$ (structure), 1656.796 (peptide mass [M]) and VCQDCPLLAPLNDTR (peptide sequence)

8. For the AB labelled $N$-linked test data the O-linked the compositions with UniCarbKB entries should be:

\begin{tabular}{|c|c|c|c|}
\hline Composition & $\begin{array}{l}\text { Submitted [M } \\
\quad-\mathbf{H}]\end{array}$ & $\begin{array}{l}\text { Glycoform } \\
\text { mass }\end{array}$ & $\begin{array}{l}\Delta \text { mass } \\
\text { (Dalton) }\end{array}$ \\
\hline $\begin{array}{l}(\text { HexNAc })_{2}(\text { Deoxyhexose })_{1}+ \\
(\mathrm{Man})_{3}(\mathrm{GlcNAc})_{2}\end{array}$ & 1581.92 & 1444.534 & 0.318 \\
\hline $\begin{array}{l}(\mathrm{Hex})_{1}(\mathrm{HexNAc})_{2}(\text { Deoxyhexose })_{1}+ \\
(\mathrm{Man})_{3}(\mathrm{GlcNAc})_{2}\end{array}$ & 1744.00 & 1606.587 & 0.345 \\
\hline $\begin{array}{l}(\mathrm{Hex})_{2}(\mathrm{HexNAc})_{2}(\text { Deoxyhexose })_{1}+ \\
(\mathrm{Man})_{3}(\mathrm{GlcNAc})_{2}\end{array}$ & 1906.01 & 1768.64 & 0.302 \\
\hline
\end{tabular}

9. For the reduced $O$-linked test data the $O$-linked the compositions with UniCarbKB entries (in addition composition that are less likely to occur in nation) should be:

\section{Composition}

$(\mathrm{Hex})_{1}(\mathrm{HexNAc})_{1}$

$(\mathrm{Hex})_{1}(\mathrm{HexNAc})_{1}(\mathrm{NeuAc})_{1}$

$(\mathrm{Hex})_{1}(\mathrm{HexNAc})_{1}(\mathrm{NeuAc})_{2}$

$(\mathrm{Hex})_{2}(\mathrm{HexNAc})_{2}(\mathrm{NeuAc})_{2}$
Submitted [M -

H]

384.1

675.1

966.2

1331.2
Glycoform mass

365.132

656.228

947.323

1312.455
$\Delta$ mass (Dalton)

$-0.05$

$-0.146$

$-0.141$

$-0.273$

\subsection{From structure to predicted MS/MS data: GlycoWorkbench}

GlycoWorkbench is designed for rapid structure drawing, theoretical fragment computation, and association with theoretical fragment with experimental MS/MS peaklist as well as other many useful functions.

1. Start GlycoWorkbench and draw the putative glycan structure. Go to Structure $>$ Add structure, and choose the type of glycan you to draw on canvas. 
The structure can be further modified by other functional tools in Structure drop down list, such as Add residue and Add terminal.

2. To set the theoretical mass, go to Tools>Mass options of all structures, where theoretical mass can be changed according to different settings.

3. To past MS/MS peaklist to GlycoWorkbench (Figure 3A), copy the list of fragments $\mathrm{m} / \mathrm{z}$ and intensity in Excel file (Note 6)

4. To calculate the theoretical fragmentation ions of drawn structure, select

Tools $>$ Fragments $>$ Compute fragments for selected structures. The window of Fragment options appears. After select glycosidic and cross-ring cleavage, click OK (Figure 3B). The theoretical fragmentation list will appear in right panel (Figure 3C).

5. To associate experimental MS/MS peaklist with computed peaklist, go to Tools $>$ Annotation $>$ Annotate peaks with fragments from selected structures. A Fragment option window will appear. After set the parameter, click OK. Then associated peaklist will appear in right panel (Figure 3D).

\subsection{From analytical MS and MS/MS data to structure: UniCarb-DB}

Different searches have been implemented to retrieve data in UniCarb-DB:

a) Taxonomy/Tissue, where entries are selected based on their biological context,

b) Mass and composition. As the name indicates, the search can be performed by mass and/or composition. More parameters can be used to make the query more specific.

c) MS/MS search, or peak matching tool, retrieves and scores the peak lists in the database according to their similarity to a target peak list. The minimum input data is a peak list on plain text separated by a tab stop character (Figure 4A). These data can be copied and pasted directly into the search page from softwares such as Microsoft Excel or directly copied from MS software.

\subsubsection{Mass and composition}

1. Upon accesing UniCarb-DB on http://www.unicarb-db.org/ click on the "Get Started" buttom at the bottom of the page.

2. Select the option "Search" from the menu located at the top of all interfaces. Afterwards, click on the option "Mass and composition" from the menu located at the left side in the search page.

3. Locate the "composition" block at the bottom of the page. Glycan composition can be assembled by selecting the option "yes" from the respective drop down list if the desired residue must be included in the structure. "no" is chosen if the residue must not be part of the structure. Possible is the default value and indicates that the residue will not be considered during the search. The text boxes following the lists are used to specify the number of residues (exact or interval) that are expected. For example, use the values " 1 ", " 1 ” and " 2 ", and " 2 " for dHex, Hex and HexNAc respectively.

4. Click on the "search" button.

5. The results show the list of structures that satisfy the constraints. The result can be refined by using the rest of parameters on the search form.

6. Return to the "Mass and composition" search form and perform all the previous steps. 
7. Input a mass value. For example, "894.3" and select the option "Calculated" (i. e.

8. The error parameter should be included only when the mass value is specified. The error is calculated for both upper and lower ranges. For example, if the value of " 0.5 " is used, the query will select all spectra whose calculated mass value lie between 894.8 and 893.8. The drop down list next to the text box includes three measurement formats for the error value: $m / z$, ppm and dalton. Leave the option selected for default $(m / z)$.

9. Select the option “-”, "underivatised” and "reduced” from the drop lists "charge state”, "derivative”, and "reducing end” respectively.

10. Click on the "search" button.

11. The number of results decrease in comparison with the previous search

\subsubsection{Peak matching tool (MS/MS search)}

1. Upon accessing UniCarb-DB on http://www.unicarb-db.org/ click on the "Get Started" bottom at the bottom of the page.

2. Select the option "Search" from the menu located at the top of all interfaces in UniCarb-DB. Afterwards, click on the option "MS/MS" from the menu located at the left side in the search page.

3. MS/MS spectra are represented as peak lists that consist of two columns separated by a tab stop character. The first column corresponds to the $m / z$ values and the second to the respective intensities. Paste the target peak list from the excel file in the proper text box in the search form (Figure 4A). This is the only requirement to start the search, however, the query can take a long time to display the results. The use of additional parameters can speed up notably the process.

4. To continue with the test case, use the value "1057.48" for the precursor mass.

5. The error parameter should be included only when the precursor mass is also being used. The error is calculated for both upper and lower ranges. For example, with an error value of " 0.5 ", the query will select all spectra whose precursor mass values lie between 1056.98 and 1057.98. The drop down list next to the text box includes three measurement formats for the error value: $m / z$, ppm and Dalton. For the test case, leave the default option $(\mathrm{m} / \mathrm{z})$.

6. The fragmentation error is used to adjust for the possible discrepancy between representations of numeric values.

7. The "method" drop list includes only one option for the time being "dot product". This indicates the type of algorithm that is used to perform the comparison.

8. The results are displayed as a list of structures, each one with a score (Figure 4B). The higher the score, the more similar the peak lists are.

\subsection{From H-NMR data to structure: Casper}

In the following the structural elucidation of a tetrasaccharide, viz., the most abundant $O$ mannosyl glycan on the glycoprotein $\alpha$-dystroglycan[PMID:22372538] is presented using components and unassigned NMR spectra as input. Given the input data (Figure 5A)

CASPER will for a structural elucidation produce a ranked list of structural suggestions based on the best fit of predicted chemical shifts to those from NMR experiment(s) (Figure 5B). Furthermore, tentative ${ }^{1} \mathrm{H}$ and ${ }^{13} \mathrm{C}$ NMR chemical shift assignments for all resonances are 
made; a schematic chemical shift map (HSQC-type) that compares experimental and predicted chemical shifts is generated. In addition, a three-dimensional structure in pdbformat can be made using the CarbBuilder program [ref IEEE 2011] in a seamless way from within the 'CASPER results menu'. A detailed step-by-step description on how to use CASPER was recently reported [PMID:25753701].

\subsection{From HPLC/UPLC/CE data to $N$-linked structure: GlycoBase}

GlycoBase (Figure 6) contains several glycan collections; all the searches can be performed by searching the entire collection, a selected subsection or glycans derived from a particular sample within a collection. You can apply different search parameters including features such as name, antennary composition (e.g., A2), GU value, particular glycan feature (e.g., sialic acid), stoichiometric search (e.g., number of hexoses), mass or by biological context (e.g., tissue name) or disease.

\subsubsection{Preliminary annotations based on undigested profile}

1. Launch GlycoBase

2. As a first approach it is recommended to search the entire collection to ensure complete coverage of glycan spectrum.

3. You can order all glycan structures based on increasing or decreasing GU value by simply clicking 'HILIC-HPLC'.

4. Locate the 'Simple Search' field and search for a GU value that you have previously obtained or from the example data set.

5. The first glycan peak in the example data (Figure 7, Table) has GU value of 7.15. By searching the database you can find that one potential glycan of the same GU value is the A2(4)G2 structure.

6. Continue searching the database for next peak with GU value of 8.06. The glycan structure with the most similar GU value is FA2G(4)2S(6)1; however it is advisable to make a note of other potential glycans with similar GU value.

7. Continue searching the database for remaining glycan peaks and write down all possibilities.

8. Sometimes for one glycan peak there are several options with potential structures of similar GU value. Preliminary annotations, obtained from the GlycoBase search can be verified with exoglycosidase digestions.

\subsubsection{Confirmation of annotations with exoglycosidase digestions}

1. Glycan sequencing by digestion with exoglycosidases is a very powerful tool to elucidate glycan structures. The enzymes may be used in a single or panel digestion array. They specifically cleave glycosidic bonds between individual monosaccharides units from the non-reducing end and are highly specific for both the terminal residue and its linkage type.

2. An example of the exoglycosidase digestion array from fibrinogen is shown in Figure 7. 
3. Based on the comparison between chromatographic profiles obtained before and after digestion with exoglycosidase of known specificity, confident assignments can be made.

4. Apply the strategy of top-down digestion and bottom-up identification.

5. Start with the first digestion, ABS (Arthrobacter ureafaciens sialidase) releases $\alpha 2-$ 3, $\alpha 2-6$ and $\alpha 2-8$ linked non-reducing terminal sialic acid residues. Observe the peaks that are eliminated with sialidase treatment. Peaks that are removed, are susceptible to sialidase treatment, thus implying they contain the sialic acid residue at the terminal end. To further validate linkage type, additional digestions with a NANI sialidase may release $\alpha 2-3$ linked non-reducing terminal sialic acid.

6. Continue evaluating the subsequent digestions and observe shifts in peaks that correspond to particular glycan residues: BKF (bovine kidney $\alpha$-fucosidase) releases $\alpha 1-2,3,4,6$ linked non-reducing terminal fucose residues, BTG (bovine testes $\beta$-galactosidase) hydrolyses non-reducing terminal $\beta 1-3$ and $\beta 1-4$ linked galactose and GUH (hexosaminidase cloned from Streptococcus pneumoniae expressed in E. coli) releases GlcNAc residues linked to Man (Figure 7).

7. Confirm potentially identified structures by checking their GU value with the database.

8. Always double check the exported table containing GU values and the corresponding relative percentage areas. Area values of species before and after a digest should match.

9. In case of complex samples it is possible to collect individual peaks, the volatile buffers can be removed and the glycans can be analyzed by mass spectrometry to validate assignments. Combination of different orthogonal techniques increases confidence of structure identification.

\subsection{Validation of monosaccharide sequence and linkages with the aid of exoglycosidase digestion: GlycoDigest}

A description of the tool including example data and details of exoglycosidases can be found at http://www.glycodigest.org. From the homepage links guide users to the 'Getting Started Guide' hosted on GitHub at http://bit.ly/1lZlcFk in addition to 'Exoglycosidase Information' options and example data. The web application version of GlycoDigest has been integrated into UniCarbKB:

\subsubsection{Step One: Build Glycan Structure}

1. Launch UniCarbKB-GlycoDigest (http://unicarbkb.org/builderDigest) (Note 7)

2. The latest version of GlycanBuilder offers the same functionality developed by EUROCarbDB project. Start building your structure by (i) selecting monosaccharides from the canvas panel or the 'Structure' menu alternatively (ii) extend a structure from a predefined list or (iii) build a substructure/epitope.

3. Structures can be built by adding new monosaccharides or pre-defined groups to the highlighted residue. New groups can be either selected from the medium toolbar or from the structures menu. The list of structural constituents comprises an exhaustive collection of saccharides, substituents, reducing-end markers and saccharide 
modifications. All the stereo-chemical information about a saccharide, like anomeric conformation, chirality, ring configuration and linkage position, can be specified.

4. After building the appropriate glycan structure select the 'Digest' Button

\subsubsection{Step Two: Selecting Exoglycosidases}

The next step is to theoretical digest the structure with a defined array of exoglycosidases. Careful design and formulation of exoglycosidase arrays is critical to the success of correct glycan structural assignments.

1. To create an array of exoglycosidases use the 'Build Exoglycosidase Array' box to select the supported exoglycosidases. (Note 8)

2. After selecting a single or panel of enzymes select the 'Digest' button.

\subsubsection{Step Three: Output}

GlycoDigest will simulate the combinations of exoglycosidase selected in Step Two. When complete the 'Results' section will be updated with theorectially computed end-product(s).

\subsection{Association of structure with biology; UniCarbKB and association structures with protein}

UniCarbKB is an initiative that provides an online information storage and search platform for glycomics and glycobiology research. The knowledgebase offers open access to a rich resource supported by querying interfaces, annotation technologies and the adoption of common standards to integrate structural, experimental and functional data.

A step-by-step guide on how-to use the database is provided (ref Kiyoko Book Chapter), however, to centralize and organize the features of UniCarbKB a confluence knowledge base is available (http://confluence.unicarbkb.org). The knowledgebase is a repository of how-to and troubleshooting guides that provides users with access to solutions and relevant search results.

The confluence knowledge base is split into four main sections: i) Content, ii) Browsing, iii) Nomenclature, and iv) How-to articles. Each section is further divided into specific layers that focus on a particular feature, which includes screenshots and step-by-step notes on discovering data sets and navigating the database.

1. Launch UniCarbKB Confluence (http://confluence.unicarbkb.org)

2. Select a section from the navigation panel on the left-hand side. For example, to find information about browsing glycoproteins click the 'Browsing UniCarbKB' link followed by 'the Glycoprotein Page'.

3. Each page summarises the content available with documented screenshots and how users can access relevant information. For the 'Glycoprotein Page' a screenshot highlights the major content sections and briefly describes the level of information available.

4. The 'Related articles' section provides links to related guides.

5. The 'Search' box offers a quick navigation aid as well as a full site search. When you perform a full search, Confluence will search all content and the result screen shows a 
list of matching items.

The 'How-to articles' provide new and returning users with access to up to date documented guides. For example, the 'Reference' guide provides an overview of selecting and searching reference and a screenshot of an individual reference page.

\subsection{Association of structure with microbial interactions: SugarBind}

Description of what will be achieved

Bullet points description of how to utilize the database

Screen capture of how to use the tool

\section{Notes}

1. The GlycoMod tool is specifically designed for $N$-linked and $O$-linked glycoproteins. The possible allowed $N$-linked compositions are more restricted by the presence of the core pentasaccharide (GlcNAc2, Man3) than the $O$-linked, so for generic searches of other types of glycoconjugates the $O$-linked search is recommended.

2. Since the database is currently mainly consisting of negative ion mode MS/MS fragment spectra, we are happy to include other type of data in the database, submitted as glycoworkbench files. If the MS/MS fragment spectra from the same structure, derivate, ion mode and charge state has not been submitted to the database, the result from the searching is probably not valid.

3. Charge deconvolution of MS data is required if the charge state of pseudomolecular ions is higher than $+/-1$.

4. For mammalian type of sugars the left hand column monosaccharides of (7) is advised be left as selection "possible", while the right hand column should be selected only if you have additional knowledge or indication that they are present in your sample. Humans usually don't have NeuGc.

5. The term "glycoforms mass" is referring to an uncharged mass of a glycan without its reducing end modification, i. e. it should not be considered as the mass of the glycan in its reducing form where addition water needs to be added

6. For computer operating system using “comma (,)" as decimal point, all comma should be changed to “dot (.)” before pasting MS/MS peak list to GlycoWorkbench. For Xcalibur, one can export the list directly using Export>Clipborad (Exact Mass) to GlycoWorkbench. When paste peaklist, try to use function of right button by highlight the first row or paste button at right bottom. Usually, Crtl+V does not work.

7. To successful model the action of exoglycosidase the monosaccharide anomeric form and glycosidic linkages must be defined. Exoglycosidases are highly-specific and the rules embedded in GlycoDigest will only work if the submitted structure is fully-defined. To specify linkage and anomeric form select a residue and use the 1st linkage link to configure from the main GlycanBuilder canvas.

8. The abbreviated names of the exoglycosidases is used, but a pop-up table is available that summarises the specificity of the exoglycosidases. To access the table select the 'Information on Exoglycosidases' button 


\section{Acknowledgements}

The authors acknowledge support from the European Union FP7 GastricGlycoExplorer ITN under grant agreement no.316929, Petrus and Augusta Hedlunds foundation, The Swedish Foundation for International Cooperation in Research and Higher Education and the Swedish Research Council (621-2013-5895).

\section{REFERENCES}

1. $\quad$ Royle, L., et al., Detailed Structural Anlysis of N-glycans Released From Glycoproteins in SDS-PAGE Gel Bands Using HPLC Combined With Exoglycosidase Array Digestions, in Methods in Molecular Biology, I.Brockhausen, Editor. 2006, Humana Press Inc.: Totowa, NJ. p. 125-143.

2. $\quad$ Royle, L., et al., HPLC-based analysis of serum N-glycans on a 96-well plate platform with dedicated database software. Anal Biochem, 2008. 376(1): p. 1-12.

3. Adamczyk, B., et al., Characterization of fibrinogen glycosylation and its importance for serum/plasma N-glycome analysis. Journal of proteome research, 2012. 12(1): p. 444-454. 


\section{Figure Captions}

Figure 1. The future of integration of -omic research in biology and medicine in order to understand the interplay between molecules of life.

Figure 2. Input page from Glycomod, showing; A, area for input of MS parameters; B, input of glycoconjugate meta data; and C, monosaccharide compositional data. Numbers refer to the text 3.1.1.

Figure 3. Annotation of experimental MS/MS peaklist using GlycoWorkbench. (A) Input experimental MS/MS pealist into GlycoWorkbench; (B-C) Computing theoretic glycosidic and cross-ring cleavage using GlycoWorkbench; (D) Associating experimental MS/MS peaklist with computed fragment of selected structure.

Figure 4. UniCarb-DB MS/MS query. A, Input page of the MS/MS search page on UniCarb$\mathrm{DB}$ and $\mathrm{B}$, result page of submitted MS/MS fragment data using normalised dot product.

Figure 5. Components, ${ }^{1} \mathrm{H}$ and ${ }^{13} \mathrm{C}$ NMR data given as input to CASPER for structural elucidation of the glycan part of a glycopeptide (A).CASPER output of the top-ranked structure for the tetrasaccharide entity corresponding to an $O$-mannosyl glycan on $\alpha$ dystroglycan (B).

Figure 6. An overview of GlycoBase components A) GlycoBase's homepage with a simple and intuitive interface B) Search tool that allows you to search for glycan structures in a number of ways $\mathrm{C}$ ) a table obtained from an example search $\mathrm{D}$ ) the description and summary for A2G2 structure. Each glycan entry contains information about properties, isomers, average GU values from different modes of separation, option to display glycan cartoon in different notation and a link to view entry in UniCarb-DB E) exoglycosidase digestions data including published digestions and internal digestions records.

Figure 7. An example data acquired from 2AB labelled glycan separation from fibrinogen containing 1) dextran ladder with indicated GU values, 2) undigested profile of fibrinogen with major peaks shown (3) sialidase digested 4) sialidase and fucosidase digested 5) sialidase, fucosidase and galactosidase digested 6) sialidase, fucosidase, galactosidase, $\mathrm{N}$ acetylhexosaminidase digested. Symbols denoted on the right of each chromatogram indicate the individual monosaccharides removed through exoglycosidase digestion. Table on the right contain detected peaks with assigned GU value and relative \% area for all peaks togeteher with annotated structure 


\section{Table Captions}

Table 1. List of databases relevant for Glycomics and Glycoproteomics

Supplementary Table 1. MS/MS peak list of a PA-labelled $N$-linked glycan with $[\mathrm{M}-\mathrm{H}]^{-}$ ions of $\mathrm{m} / \mathrm{z} 1311.08$ and a composition of 5 hexoses and $2 \mathrm{~N}$-acetylhexosamines

Supplementary Table 2. MS/MS peak lists of a reduced $N$-linked oligosaccharide with of [M $-\mathrm{H}]$ ion of $\mathrm{m} / \mathrm{z} 1056.48$ 
Tables

Table 1

\section{SCIENCE DATABASES THAT INCLUSIVELY CONTAIN} GLYCOINFORMATION

The Universal Protein Resource (UniProt) is a resource for protein sequence with annotations, www.uniprot.org

Glycodatabase related: Predicted and confirmed sites of glycosylation within protein amino acid sequences

References: Farriol-Mathis et al (PMID15174124)

The Protein Data Bank (PDB) is a database three-dimensional data of large biological molecules, such as proteins and nucleic acids, www.rcsb.org

Glycodatabase related: Information about localisation of $N$-and $O$-linked glycosylation sites as well as ligand localisation of carbohydrate binding proteins.

References: Berman et al PMID 10592235

The Gene Expression Omnibus (GEO) gene expression data repository, www.ncbi.nlm.nih.gov/geo

Glycodatabase related: Expression profiles of glycorelated genes in cell models, animal models and clinical samples

Reference: Barett et al PMID 23193258

The Human Proteome Atlas is a tissue, cell line, and cancer tissue map of the proteome, www.proteinatlas.org

Glycodatabase related: Glycoprotein and glycogene proteins localisation in tissues an subcellular localisation

References: Uhlén et al PMID 25613900

LipidBank is a database of natural lipids, lipidbank.jp

Glycodatabase related: Literature data and experimental evidence (eg MS, UV, IR, NMR) of glycolipids

References: Watanabe K., Yasugi E. and Oshima M. "ref How to search the glycolipid data in LIPIDBANK for Web: the newly developed lipid database" Japan Trend Glycosci. and Glycotechnol. 12, 175-184, 2000. PMID

LIPID MAPS contains chemical repressentation and annotations of biologically relevant lipids, www.lipidmaps.org/

Glycodatabase related: Chemical representations of glycoplipids form consortium and literature data

Reference: Sud et al (PMID 17098933)

EXCLUSIVE GLYCODATABASES

The Consortium for Functional Glycomics (CFG) is an international initiative providing glycomic services and databases, www.functionalglycomics.org

Glycodatabase related: Data from the screening of the consortiums glycan array screening, glycogene microarray of tissues and cells, MALDI-MS screening of glycans from mouse and human tissue. It also contains information about histology, immunology, hematology and metabolism/behaviour of mice knockouts with glyco-related genes. The "CFG Molecule Pages” integrates the data generated from the CFG screening together with public data about glycosyltransferases, glycan structures and glycan binding proteins.

Glycan array data from the consortium can be mined by GlycoPattern

(glycopattern.emory.edu) (PMID 25143288)

The UniCarb KnowledgeBase (UniCarbKB) is a curated database of information on the glycan structures of glycoproteins reported in scientific literature, www.unicarbkb.org 
Glycodatabase related: Current version contain 3238 structures from the literature including information about taxonomy, tissue and associated protein

Reference: Campbell et al (PMID 24234447)

The UniCarb-DB database is an assembly of glycosequences with associated MS fragmentation data, www.unicarb-db.org

Glycodatabase related: Current version contain more than 600 spectra from published work including information about structure, taxonomy, tissue and associated protein Reference: Hayes et al (PMID 21398669)

GlycomeDB is a metadatabase of carbohydrate sequences, www.glycome-db.org

Glycodatabase related: Database include structures from CFG , KEGG,

GLYCOSCIENCES.de, BCSDB and Carbbank (ref), including information about species and associated aglycon

References: Ranzinger et al PMID 21045056

GlyTouCan, International glycan sequence repository, glytoucan.org

Glycodatabase related: Registration of glycan structures with minimal metadata to obtain a unique accession number.

References: Aoki-Kinoshita et al PMID 26476458

Kyoto Encyclopedia of Genes and Genomes (KEGG) is a database of genome sequences and other high-throughput data, www.genome.jp/kegg

Glycodatabase related: www.genome.jp/kegg/glycan. Pathways for glycan biosynthesis and metabolism, roles of glycans in cellular processes, glycans in cancer, glycosyltransferase reactions and relation to diseases, carbohydrate binding proteins, The KEGG GLYCAN structure database contains structures from CarBank (ref), literature, and from structures present in KEGG pathways.

Reference: Kanehisa et al PMID 26476458, Hashimoto et al (PMID 16014746)

The Japan Consortium for Glycobiology and Glycotechnology DataBase (JCCGB) is a metdadatabase that allow you to simultaneously search in glyco related databases, jcggdb.jp Glycodatabase related: The current version include GGDB (GlycoGene Database, jcggdb.jp/rcmg/ggdb), LfDB (Lectin Frontier Database, jcggdb.jp/rcmg/glycodb/LectinSearch), GlycoPOD (Glyco-Science Protocol Online Database, jcggdb.jp/GlycoPOD), GlycoProtDB (GlycoProtein Database, jcggdb.jp/rcmg/gpdb), GMDB (Glycan Mass Spectral Database, jcggdb.jp/rcmg/glycodb/Ms_ResultSearch), LipidBank, GlycoEpitope, GALAXY (Glycoanalysis by the three axes of MS and chromatography), KO mice DB (Functional Glycomics with KO Mice Database, metadb.riken.jp/metadb/db/Glycomics_mouse), Glycoforum, PACDB (Pathogen Adherence to Carbohydrate Database, jcggdb.jp/search/PACDB.cgi, GDGDB (Glyco-Disease Genes Database), TuMaRdb (Tumor Markers Reference Database), JCGGDB Reports, GlycosidaseDB, and JIGSDB (JCGG Integrated Glycan Structures Database).

Reference: Maeda et al (PMID 25753710)

The GlycoEpitope is a database of 173 common name of glycobiological epitopes associated with a know oligosaccharide sequence, www.glycoepitope.jp

GALAXY is a database of glycan sequences Glycoanalysis by the three axes of MS and chromatography, www.glycoanalysis.info

Glycodatabase related: Elution profiles of PA labelled $N$-linked oligosaccharide in by different LC-methods

Reference: Tomiya et al PMID 3407923

GlycoDomain Viewer is a graphic interface that displays a database of $O$-linked and $N$ linked glycosylation sites on proteins based on UniProt annotation and in-house discovery, glycodomain.glycomics.ku.dk 
Glycodatabase related: Database of $O$-linked sites has been used in the NetOGlyc neural network predictions of mucin type GalNAc O-glycosylation sites in mammalian proteins (www.cbs.dtu.dk/services/NetOGlyc)

Reference: Steentoft et al PMID 23584533

GLYCOSCIENCES is a carbohydrate sequence database from literature with taxonomy, linking to PDB, NMR and 3D modules, glycosciences.de

Glycodatabase related: Database built on Carbank (ref) and is updated by including glycans from PDB. It also includes the MonosaccharideDB, a database of monosaccharides (www.monosaccharidedb.org)

Reference: Lütteke et al PMID 16239495

Glygobase (Lille) is a glycan sequence database with taxonomy and NMR, glycobase.univlille1.fr/base

Glycobase (Dublin) is a database with glycan sequences including HPLC experimental data, taxonomy, MS and literature references.

Glycodatabase related: Current version v3.2 include 655 sequences

Reference: Campbell et al (PMID18344517)

Carbohydrate Structure Database (CSDB) is a merged database of Bacterial (BCSDB)

and Plant and Fungal (PFCSDB) databases, csdb.glycoscience.ru

Glycodatabase related: Structures of glycans and NMR recorded in literature

Glyco3D is a portal for databases covering the three-dimensional features of

monosaccharides, disaccharides, oligosaccharides, polysaccharides, glycosyltransferases,

lectins, monoclonal antibodies against carbohydrates, and glycosaminoglycan-binding proteins, www.glyco3d.cermav.cnrs.fr

Glycodatabase related: The database contains 3 dimensional data of oligosaccharides (BiOligo), Lectins (Lectin3D), Glucosaminoglycan binding proteins with ligands (GAG), Monoclonal antibodies with the carbohydrate ligands (mAbs), glycosyltransferases (GT) and polysaccharides (Polysac).

Reference: Pérez et al (PMID 25753716)

The Carbohydrate-Active enZYmes Database (CAZy) contains genomic, structural and biochemical information on glyco-enzymes, www.cazy.org

Reference: Lombard et al (PMID 24270786)

The Unipep database contains theoretical N-linked glycolylation sites in UniProt that have been mapped to LC-MS data, www.unipep.org

Reference: Zhang et al (PMID 16901351)

GLYCAM libraries contain predicted 3D-structures of oligosaccharides. Structures are available for $N$-linked, $N$-acetyllactosamine building blocks, and from the CFG-array, glycam.org/Pre-builtLibraries.jsp

Reference: Kirschner et al (PMID 17849372)

sugarbind

Glycodatabase related: Frederique

Reference:

nextprot

Glycodatabase related: Frederique

Reference:

ECODAB (Escherichia coli O-antigen database) contains O-polysaccharide structures of E. coli lipopolysaccharides, putative, predicted or confirmed glycosyltransferase functions, ${ }^{1} \mathrm{H}$ and ${ }^{13} \mathrm{C}$ NMR chemical shift data, flippase and polymerase sequences and literature references, www.casper.organ.su.se/ECODAB/

Reference: Rojas-Macias et al (PMID:25352573) 
EK3D is a manually curated database of K antigens corresponding to various $E$. coli serotypes and contains also their modelled 3D structures, www.iith.ac.in/EK3D/

Reference: Reddy Kunduru et al (PMID:26615200)

Supplementary Table 1

\begin{tabular}{|cc|}
\hline $\boldsymbol{m} / \mathbf{z}$ & Int \\
\hline 383.1 & 1.1 \\
\hline 406.98 & 1 \\
\hline 466.7 & 0.3 \\
\hline 467.14 & 0.8 \\
\hline 482.85 & 1.3 \\
\hline 484.97 & 18.3 \\
\hline 501.27 & 0.8 \\
\hline 503.09 & 14 \\
\hline 540.94 & 0.8 \\
\hline 545 & 25.5 \\
\hline 559.23 & 1.6 \\
\hline 574.89 & 11.8 \\
\hline 583.2 & 0.4 \\
\hline 587.02 & 1.2 \\
\hline 599.11 & 5.3 \\
\hline 599.36 & 2.2 \\
\hline 602.91 & 0.7 \\
\hline 604.28 & 0.7 \\
\hline 611.05 & 0.7 \\
\hline 614.9 & 0.4 \\
\hline 620.97 & 0.5 \\
\hline 628.94 & 30.4 \\
\hline 641.18 & 0.4 \\
\hline 647.13 & 27.9 \\
\hline 663.11 & 0.1 \\
\hline 664.51 & 1.2 \\
\hline 664.96 & 0.7 \\
\hline 670.98 & 1.5 \\
\hline 687.96 & 0.8 \\
\hline 703.12 & 1.3 \\
\hline 706.41 & 0.3 \\
\hline 707.12 & 20.4 \\
\hline 708.86 & 2.4 \\
\hline 712.93 & 1.7 \\
\hline 720.97 & 1.8 \\
\hline 730.9 & 1.1 \\
\hline 736.13 & 0.5 \\
\hline 748.22 & 1.2 \\
\hline 748.82 & 2.6 \\
\hline 766.87 & 0.7 \\
\hline 775.88 & 1 \\
\hline 789.15 & 0.9 \\
\hline & \\
\hline
\end{tabular}




\begin{tabular}{|cc|}
\hline 806.93 & 2.3 \\
\hline 809.17 & 1 \\
\hline 824.97 & 6 \\
\hline 827.01 & 2 \\
\hline 831.79 & 2.3 \\
\hline 849.56 & 2.6 \\
\hline 850 & 9.8 \\
\hline 850.28 & 3.4 \\
\hline 865.09 & 1 \\
\hline 868.01 & 0.6 \\
\hline 868.99 & 201.1 \\
\hline 872.76 & 0.5 \\
\hline 882.7 & 0.2 \\
\hline 883.18 & 0.5 \\
\hline 889.23 & 1.3 \\
\hline 893.15 & 0.9 \\
\hline 910.19 & 0.2 \\
\hline 910.99 & 13.8 \\
\hline 928.03 & 1.6 \\
\hline 929.15 & 0.4 \\
\hline 932.82 & 2.3 \\
\hline 934.14 & 1.5 \\
\hline 969.2 & 1.2 \\
\hline 981.59 & 1.3 \\
\hline 987.17 & 2.2 \\
\hline 993.91 & 2 \\
\hline 999.44 & 0.6 \\
\hline 1010.84 & 1.3 \\
\hline 1011.98 & 93.8 \\
\hline 1015.15 & 0.2 \\
\hline 1026.17 & 1 \\
\hline 1047.03 & 1.9 \\
\hline 1053.49 & 2.9 \\
\hline 1071.04 & 2.5 \\
\hline 1071.83 & 0.6 \\
\hline 1072.14 & 0.6 \\
\hline 1089.12 & 10.9 \\
\hline 1106.58 & 1.4 \\
\hline 1107.15 & 6.1 \\
\hline 1131.16 & 8.3 \\
\hline 1131.54 & 1 \\
\hline 1132.29 & 0.7 \\
\hline 1134.89 & 1.3 \\
\hline 1149.01 & 150.4 \\
\hline 1190.59 & 0.4 \\
\hline 1190.98 & 4.8 \\
\hline 1191.27 & 1 \\
\hline 1198.14 & 0.8 \\
\hline & \\
\hline
\end{tabular}




\begin{tabular}{|cc|}
\hline 1207.92 & 1.2 \\
\hline 1209 & 10.5 \\
\hline 1209.27 & 2.7 \\
\hline 1227.06 & 0.6 \\
\hline 1233.04 & 0.8 \\
\hline 1251.01 & 150 \\
\hline 1269.04 & 73.4 \\
\hline 1292.97 & 12.8 \\
\hline 1293.37 & 0.5 \\
\hline 1311.07 & 2.1 \\
\hline
\end{tabular}

Supplementary Table 2

\begin{tabular}{|cc|}
\hline $\mathbf{m} / \mathbf{z}$ & Int \\
\hline 305.07 & 38.2 \\
\hline 308.2 & 8.1 \\
\hline 323.06 & 88.4 \\
\hline 324.06 & 7.7 \\
\hline 341.03 & 21.1 \\
\hline 350.16 & 350.7 \\
\hline 351.16 & 35.7 \\
\hline 365.09 & 9 \\
\hline 368.17 & 78.2 \\
\hline 369.17 & 11.2 \\
\hline 383.13 & 180.5 \\
\hline 384.14 & 22.2 \\
\hline 389.18 & 31.4 \\
\hline 407.14 & 53.4 \\
\hline 408.11 & 10.4 \\
\hline 425.26 & 7.5 \\
\hline 449.23 & 6.8 \\
\hline 503.07 & 37.3 \\
\hline 508.24 & 6.7 \\
\hline 511.21 & 21 \\
\hline 526.18 & 157.3 \\
\hline 527.16 & 54.5 \\
\hline 528.2 & 7.6 \\
\hline 529.21 & 8.1 \\
\hline 545.17 & 1043.5 \\
\hline 546.19 & 169.6 \\
\hline 553.2 & 295.8 \\
\hline 554.19 & 47 \\
\hline 559.19 & 22.1 \\
\hline 569.25 & 9.2 \\
\hline 570.22 & 16 \\
\hline 571.21 & 81.9 \\
\hline 572.23 & 19.5 \\
\hline 586.27 & 7.4 \\
\hline 587.1 & 80.9 \\
\hline & \\
\hline
\end{tabular}




\begin{tabular}{|cc|}
\hline 588.08 & 10 \\
\hline 600.2 & 20.3 \\
\hline 601.24 & 10.6 \\
\hline 605.13 & 9.3 \\
\hline 629.23 & 9.1 \\
\hline 647.24 & 7 \\
\hline 671.23 & 46.8 \\
\hline 672.1 & 8.3 \\
\hline 672.37 & 6.8 \\
\hline 687.06 & 7.2 \\
\hline 688.22 & 681.1 \\
\hline 689.21 & 285.6 \\
\hline 690.24 & 35.1 \\
\hline 691.24 & 8.3 \\
\hline 713.22 & 15.3 \\
\hline 731.24 & 277.3 \\
\hline 732.23 & 130.3 \\
\hline 733.29 & 48.5 \\
\hline 734.31 & 6.6 \\
\hline 748.07 & 10.4 \\
\hline 749.22 & 25.9 \\
\hline 750.31 & 6.4 \\
\hline 762.28 & 51.7 \\
\hline 763.34 & 11.3 \\
\hline 809.36 & 8.5 \\
\hline 820.36 & 7.2 \\
\hline 833.26 & 123.7 \\
\hline 834.37 & 31.5 \\
\hline 835.27 & 107.7 \\
\hline 836.31 & 35.2 \\
\hline 851.3 & 358.2 \\
\hline 852.3 & 106.9 \\
\hline 853.29 & 191.1 \\
\hline 854.26 & 67.7 \\
\hline 877.29 & 56.9 \\
\hline 878.3 & 19.3 \\
\hline 893.27 & 848.5 \\
\hline 894.28 & 266.4 \\
\hline 895.27 & 1496.8 \\
\hline 896.29 & 698.5 \\
\hline 897.29 & 65.3 \\
\hline 911.31 & 70.2 \\
\hline 912.27 & 188.3 \\
\hline 913.26 & 46 \\
\hline 924.18 & 13.9 \\
\hline 924.42 & 8.6 \\
\hline 925.26 & 55.1 \\
\hline 926.24 & 20 \\
\hline & \\
\hline
\end{tabular}




\begin{tabular}{|cc|}
\hline 936.43 & 7 \\
\hline 937.23 & 66.2 \\
\hline 938.29 & 18 \\
\hline 954.1 & 10.5 \\
\hline 955.32 & 31.1 \\
\hline 956.38 & 10.3 \\
\hline 973.19 & 7.4 \\
\hline 997.3 & 325.6 \\
\hline 998.3 & 144.9 \\
\hline 1008.11 & 9.2 \\
\hline 1008.38 & 7.8 \\
\hline 1014.32 & 6.7 \\
\hline 1015.3 & 756.9 \\
\hline 1016.31 & 312.9 \\
\hline 1020.29 & 10.1 \\
\hline 1021.8 & 13.5 \\
\hline 1022.35 & 6.9 \\
\hline
\end{tabular}

